\title{
Evidence of Passive Smoking as a Risk Factor of High-Grade Squamous Intraepithelial Lesion: A Case-Control Study
}

\author{
Xinxin Du, ${ }^{a, b, \#}$ Mingxia Li, ${ }^{a, \#}$ Yi Zhou, ${ }^{c}$ Hao Yang, ${ }^{d}$ Vladimir Isachenko, ${ }^{b}$ Tatsuya Takagi, ${ }^{*, c}$ and \\ Yuanguang Meng*,d \\ ${ }^{a}$ Department of Obstetrics and Gynecology, Chinese PLA General Hospital; 28 Fuxing Rd., Haidian District, Beijing \\ 100853, China: ${ }^{b}$ Department of Obstetrics and Gynecology, Medical Faculty, University of Cologne; Kerpener \\ Street 34, Cologne 50931, Germany: ${ }^{c}$ Laboratory of Pharmainformatics and Pharmacometrics, Graduate School of \\ Pharmaceutical Sciences, Osaka University; 1-6 Yamadaoka, Suita, Osaka 565-0871, Japan: and Department of \\ Radiation Oncology, Inner Mongolia Cancer Hospital and The Affiliated People's Hospital of Inner Mongolia Medical \\ University; Hohhot 010020, China.
}

Received December 19, 2019; accepted April 9, 2020

This study aims to evaluate the association between passive smoking and high-grade squamous intraepithelial lesion (HSIL) at the sample of Chinese women. We conducted a case-control study to analyze the effect of passive smoking on the incidence that patients diagnosed with HSIL. The participants had undergone cervical cancer screening by cytology and human papillomavirus (HPV) co-testing within a year before the study. Multiple logistic regression was used to explore the effect and interactive effect of risk factors on HSIL. The odds ratio (OR) and 95\% confidence interval (CI) were calculated. Passive smokers were 1.57 times (95\% CI 1.05-2.35) higher than non-smokers to occur HSIL. The medium of the combined smoking index divided patients into low and high exposure, with the ORs of 1.64 (95\%CI 1.02-2.64) and 1.71 (95\%CI 1.06-2.77) relative to non-smokers, respectively. The combined smokers in the high exposure group experienced the most considerable risk of HSIL $(\mathrm{OR}=4.67 ; 95 \% \mathrm{CI} 1.17-18.70)$. The OR of HPV positive passive smoker relative to that of HPV negative non-smokers was 5.28 (95\%CI 2.25-14.52;). Passive smokers who reported adolescent exposure history was 4.04 times $(95 \%$ CI $1.44-11.37)$ more at risk of the disease than non-smokers. This study supported that passive smoking was a significant independent risk factor on the occurrence of HSIL and showed a positive correlated dose-response relationship. HPV infection interacting with passive smoking led to an even higher risk of the disease. Adolescent exposure to passive smoking persistent for more than 20 years would also increase the risk of HSIL.

Key words passive smoking; high-grade squamous intraepithelial lesion; human papillomavirus; adolescent exposure

\section{INTRODUCTION}

Cervical cancer is the fourth most frequently diagnosed cancer worldwide, and the second in less-developed countries. In 2012, a total of 444500 newly diagnosed cases and a crude incidence rate of 15.7/100000 was reported. ${ }^{1)}$ In China, it was the fifth most common cancer in the female with 98500 newly diagnosed cases and a crude incidence rate of 14.93/100000, leading to severe social burden of this disease. ${ }^{2)}$

Several articles have reported the association between risk factors and occurrence or development of cervical intraepithelial lesions, such as persistent infection with high-risk human papillomavirus (HPV), multiple sexual partners and smoking. ${ }^{3-6)}$ However, the effect of passive smoking on the disease is unclear. Passive smoking is considered to be a common carcinogen of multiple types of cancer. ${ }^{7)}$ It is defined as environmental tobacco smoke, referring to the substance released from the smog of the lighting cigarette. In China, the passive smoking rate in recent years among women has been found significantly higher than that among men. ${ }^{8)}$ Aiming to evaluate the effect between passive smoking and cervical precancerous lesion among Chinese women, we conducted this casecontrol study. In this study, we reported the design and results

\footnotetext{
\#These authors contributed equally to this work.
}

according to the Strengthening the Reporting of Observational Studies in Epidemiology (STROBE) statement.

\section{MATERIALS AND METHODS}

Study Participants The cases were enrolled at the People's Liberation Army General Hospital, Beijing, China from October 2017 to June 2018. Potential cases were outpatients with high-grade squamous intraepithelial lesion (HSIL), ${ }^{9}$ diagnosed by cytology-HPV contesting, colposcopy and cervical biopsy. Liquid-based cytology test was classified by the 2001 Bethesda System. ${ }^{10)}$ Multi-point cervical biopsy was performed on suspected lesions under colposcopy for histopathology diagnosis. We included participants aged between 21 and 65 with detected HSIL for the first time. Exclusion criteria included: (1) history of autoimmune diseases, HSIL, cervical cancer, or other cancers; (2) pregnancy or in the postpartum period; and (3) cytology or HPV test in other hospitals within two weeks or cervical biopsy and treatments within three months. A total of 184 eligible cases were identified, and 182 were interviewed, with a response rate of $98.9 \%$. Two patients refused to participate and missed in the interview.

Controls were recruited from the same hospital in the same period of cases. Eligible criteria for controls were the same with cases except that within a year (1) obtained cytology-HPV con-

* To whom correspondence should be addressed. e-mail: ttakagi@phs.osaka-u.ac.jp; meng6512@yahoo.com 
testing with non-neoplastic diseases, or (2) low-grade squamous intraepithelial lesion (LSIL) was validated by histopathology. Non-neoplastic diseases included acute and chronic gynecological inflammation, uterine fibroids, ovarian cysts, urinary tract infection, and benign digestive system diseases. We interviewed 263 among 276 identified controls. The response rate was 95.3\%.

Data Collection and Validation The qualified researchers interviewed the participants face to face by using a structured questionnaire to collect medical information, which included age, marital status, education level, age at first pregnancy, age at first live birth, multiple sexual partners, and tobacco exposure history.

The smoking survey data were divided into two sections, one about the interviewees and the other about the source of passive smoking. The two sections involved identical questions regarding smoking history. The relevant data comprised the age at which smoking began, types of tobacco used, the average number of cigarettes smoked per day, and the number of years smoked. The place where passive smoking occurred and the identity of the smokers were recorded (i.e., at home, working place, or public area from the spouse, other family members, colleagues, or others). The number of cigarettes and years smoked referred to the period when the participant was staying with the smokers.

To assess the validity of demographics and smoking-related data, two trained doctors randomly re-interviewed 200 participants via telephone one month later (100 for each doctor). The doctors were uninformed by the previous data. After the as- sessment, the data were input by two individuals and doublechecked by a third researcher to ensure accuracy and integrity.

Smoking Exposure According to the collected information, the participants were classified into four types of tobacco exposure: (1) active; (2) passive; (3) combined; and (4) no exposure. Active exposure represented the participant was a current cigarette smoker. Passive exposure indicated a nonsmoker inhaling smokes from burning tobacco. Combined exposure was defined as a current smoker with inhalation of smokes. No exposure implied no active and passive tobacco smokes breathing in.

The exposure amount of (1) active exposure was measured by using the smoking index $(\mathrm{SI})^{11)}$ : $\mathrm{SI}=n$ cigarettes smoked per day $\times$ number of years smoked. Referring to SI, passive smoking index (PSI) was used to define the exposure amount of (2) passive exposure: PSI $=n$ cigarettes exposed per day $\times$ number of years exposed. To measure the exposure amount of (3) combined smoking, we defined combined smoking index (CSI): $\mathrm{CSI}=\mathrm{SI}+\mathrm{PSI}$. Median of CSI $\left(\mathrm{CSI}_{\mathrm{m}}\right)$ was used to distinguish low exposure with $\mathrm{CSI} \leq \mathrm{CSI}_{\mathrm{m}}$ and high exposure with $\mathrm{CSI}>\mathrm{CSI}_{\mathrm{m}}$.

HPV Testing and Pathological Diagnosis Cervical swabs samples were taken by qualified doctors and were subject to DNA extraction and real-time PCR system based on the fluorogenic 5'nuclease assay and TaqMan technique (Liferiver, Shanghai, China) that detects HPV 16, 18, 31, 33, $35,39,45,51,52,56,58,59,66$, and 68 genotypes separately. The technicians that detected HPV infection were blinded to

Table 1. Baseline Characteristics of the Participants in the Study

\begin{tabular}{|c|c|c|c|c|}
\hline Variables & $\begin{array}{c}\text { Cases } \\
N=182 \mathrm{~N}(\%)\end{array}$ & $\begin{array}{c}\text { Controls } \\
N=263 \mathrm{~N}(\%)\end{array}$ & $\begin{array}{c}\text { Total } \\
N=445 \mathrm{~N}(\%)\end{array}$ & $p$ Value $^{a)}$ \\
\hline Age (years); mean \pm S.D. & $39.37 \pm 8.24$ & $40.16 \pm 9.23$ & $39.83 \pm 8.84$ & $0.346^{b)}$ \\
\hline \multicolumn{5}{|l|}{ Age group } \\
\hline$<30$ years & $22(12.09)$ & $39(14.83)$ & $61(13.72)$ & \multirow[t]{4}{*}{$0.018 *$} \\
\hline $30-39$ years & $71(39.01)$ & $91(34.60)$ & $162(36.40)$ & \\
\hline $40-49$ years & $70(38.46)$ & $79(30.04)$ & $149(33.48)$ & \\
\hline$>49$ years & $19(10.44)$ & $54(20.53)$ & $73(16.40)$ & \\
\hline \multicolumn{5}{|l|}{ Marital status } \\
\hline Unmarried & $13(7.14)$ & $20(7.60)$ & $33(7.42)$ & \multirow[t]{2}{*}{1.00} \\
\hline Married & $169(92.86)$ & $243(92.40)$ & $412(92.58)$ & \\
\hline Age of first pregnancy; mean \pm S.D. & $23.88 \pm 3.43$ & $24.38 \pm 2.95$ & $24.18 \pm 3.16$ & $0.133^{b)}$ \\
\hline Age of first live birth; mean \pm S.D. & $25.51 \pm 3.82$ & $25.81 \pm 3.51$ & $25.69 \pm 3.64$ & $0.460^{b)}$ \\
\hline \multicolumn{5}{|l|}{ Education level ${ }^{c)}$} \\
\hline Low & $13(7.14)$ & $14(5.32)$ & $27(6.07)$ & \multirow[t]{3}{*}{0.665} \\
\hline Middle & $71(39.01)$ & $110(41.83)$ & $181(40.67)$ & \\
\hline High & $98(53.85)$ & $139(52.85)$ & $237(53.26)$ & \\
\hline \multicolumn{5}{|l|}{ Multiple sexual partners } \\
\hline No & $170(93.41)$ & $253(96.20)$ & $423(95.06)$ & \multirow[t]{2}{*}{0.266} \\
\hline Yes & $12(6.59)$ & $10(3.80)$ & $22(4.94)$ & \\
\hline \multicolumn{5}{|l|}{ HPV infection } \\
\hline No & $17(9.34)$ & $70(26.62)$ & $87(19.55)$ & \multirow[t]{2}{*}{$<0.001 *$} \\
\hline Yes & $165(90.66)$ & $193(73.38)$ & $358(80.45)$ & \\
\hline \multicolumn{5}{|l|}{ Smoking exposure } \\
\hline No exposure & $59(32.42)$ & $118(44.87)$ & $177(39.78)$ & \multirow[t]{5}{*}{$0.013^{*}$} \\
\hline \multicolumn{4}{|l|}{ Smoking exposed } & \\
\hline Passive smoking & $107(58.79)$ & $136(51.71)$ & $243(54.60)$ & \\
\hline Active smoking & $8(4.40)$ & $5(1.90)$ & $13(2.92)$ & \\
\hline Combined smoking & $8(4.40)$ & $4(1.52)$ & $12(2.70)$ & \\
\hline
\end{tabular}

S.D., standard deviation; HPV, human papillomavirus. $a$ ) Pearson's $\chi^{2}$ test. $b$ ) Student's $t$-test. $c$ ) High, university or higher; middle, secondary or tertiary; low, no formal education or primary. ${ }^{*}$ Statistical significance. 
the smoking status and cervical biopsy result of the patients.

Histopathology was taken as the diagnostic gold standard. Hematoxylin-eosin staining and immunohistochemistry were both performed for cell morphology analysis and detection of p16 and $K_{\mathrm{i}}-67$ expression. The two-tiered terminology system of high-grade and low-grade squamous intraepithelial lesions was used for diagnosing cervical precancerous lesions instead of the three-tiered cervical intraepithelial neoplasia (CIN) system. $^{12,13)}$ The reporting pathologists were blinded to the smoking status and HPV testing result of the patients. A second pathologist validated the biopsy slides of all patients before releasing a definitive report. A third pathologist was required to give the final diagnosis if inconsistency existed.

Statistical Analysis Sample size of this study was calculated by using OpenEpi 3.01 (http://www.openepi.com). We set the percent of controls exposed as $50 \%{ }^{14)}$ and odds ratio (OR) equal to 2 in the balanced unmatched case-control study. After the collection of medical information from the participants, Kappa statistics were calculated to measure the agreement of reproducibility in the data collections. The average of two records was adopted if a difference existed. The differences in the baseline demographics were examined by using Student's $t$-test for continuous variables or $\chi^{2}$ test for categorical variables. The associations between HSIL and the risk factors were measured by OR with $95 \%$ confidence interval (CI). Multiple logistic regression was used to evaluate the association and interaction effects between HSIL and risk factors. The difference between the medians was examined by using the Wilcoxon signed-rank test. Statistical significance was defined as $p<0.05$. All statistical analyses were conducted by R version 3.5.2 (https://www.r-project.org/).

Ethics Statement The ethical committee of the People's Liberation Army General Hospital approved the study (Approval number S2014-090-01). All participants signed informed consent forms before the interview.

\section{RESULTS}

Demographics The sample size was calculated to be 296 in total (148 cases and 148 controls). Finally, a total of 445 participants (182 cases and 263 controls) were enrolled in the study, including 177 no exposed patients, 243 passive smokers, 13 active smokers, and 12 combined smokers. Excellent reproducibility of age, marital status, and education level was found with Kappa statistics $=1.00$. A satisfying agreement was found in smoking exposure (Kappa statistics $=0.80$ ).

The age between cases (mean \pm standard deviation,

Table 2. The Odd Ratio between Smoking Exposures and Non-smokers on HSIL

\begin{tabular}{lcccc}
\hline \hline \multicolumn{5}{c}{ (A) No exposed and smoking involved } \\
\hline & Cases & Controls & OR & $p$ \\
\hline Smoking exposed & 123 & 145 & 1.70 & 0.009 \\
No exposure & 59 & 118 & & \\
\hline
\end{tabular}

(B) No exposed and passive smoking

\begin{tabular}{lrccc} 
& Cases & Controls & OR & $p$ \\
\hline Passive smoking & 107 & 136 & & \\
No exposure & 59 & 118 & 1.57 & 0.027 \\
\hline
\end{tabular}

$39.37 \pm 8.24)$ and controls $(40.16 \pm 9.23)$ had no significant difference in the baseline. Variations were seen in the proportions of marital status, education level, and multiple sexual partners, but no statistical significance was detected among different categories. Smoking exposure, HPV infection and age groups showed statistical significance between case and control groups (Table 1).

The Association between Passive Smoking and HSIL As shown in Table 1, passive smokers accounted for $58.79 \%$ in cases and $51.71 \%$ in controls. The OR in Table 2(A) indicated that all the exposed participants were 1.70 times $(95 \%$ CI 1.14-2.52) more likely to occur HSIL than no exposed. To evaluate the OR between passive smokers and non-smokers, we excluded 25 active and combined smokers. Among the remaining 420 participants, the OR of the passive smokers to develop HSIL was 1.57 (95\% CI 1.05-2.35) relative to that of non-smokers (Table 2(B)).

Dose-Response Relationship The ranges of CSI in case and control groups were between 1 and 1200 and had no significant differences between the two groups $(p=0.1936$, Wilcoxon signed-rank test). The $\mathrm{CSI}_{\mathrm{m}}$ was calculated to be 47 , dividing 268 smokers into low exposure $(\mathrm{CSI} \leq 47)$ and high exposure (CSI > 47). The no-exposure group was set as the reference of the odds ratios. Figure 1 displayed the dose-response relationship between the level of the smoking characteristic and HSIL risk. Passive, active and combined smoking all lead to higher risk of the incidence of HSIL, compared with no exposure. In passive and combined smokers, the risk increased more in high exposure groups than in low exposure groups. The OR in passive smokers changed from 1.64 (95\% CI 1.02-2.64) for low exposure to 1.71 (95\% CI 1.06-2.77) for high exposure, relative to that of the no exposed. The combined smokers in high exposure group showed the most considerable risk of HSIL (OR $=4.67 ; 95 \%$ CI 1.17-18.70), suggesting a combined adverse effect of both active and passive smoking on HSIL.

Smoking Exposure, HPV, and Age Group on HSIL Since smoking exposure, HPV, and age group showed significant differences between HSIL case and control groups (Table 1), we added these three factors in the multiple logistic regression. As shown in Table 3(A), the OR of passive smoking compared to that of no exposure was 1.62 (95\% CI 1.06-2.44) and that of HPV positive compared to negative was $3.32(95 \%$ CI 1.90-6.09). The OR of age groups were not statistically significant.

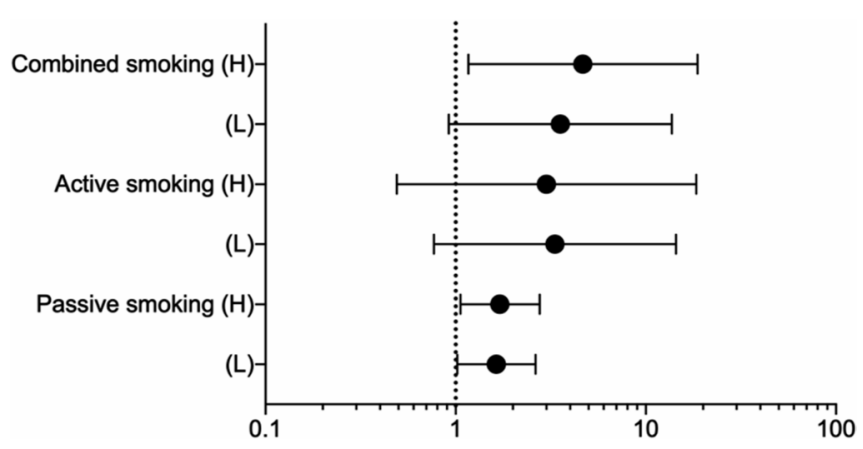

Fig. 1. Odds Ratio Forest Plot for HSIL in Patients with Different Forms of Tobacco Exposure versus No Exposure (Reference: the Noexposure Group, Odds Ratio $=1.0$ )

HSIL: high grade squamous intraepithelial lesion; L: low-level exposure; H: high-level exposure. 
Table 3. The OR and $95 \%$ CI of Risk Factors on HSIL in the Multiple Logistic Models

\begin{tabular}{|c|c|}
\hline \multicolumn{2}{|c|}{$\begin{array}{c}(\mathrm{A}) \\
(N=445)\end{array}$} \\
\hline \multicolumn{2}{|l|}{ Smoking exposure } \\
\hline No exposure & $(-)$ \\
\hline Passive smoking & $1.62 *(1.06,2.44)$ \\
\hline \multicolumn{2}{|c|}{ Passive smoking $\times<30$ years } \\
\hline \multicolumn{2}{|c|}{ Passive smoking $\times 30-39$ years } \\
\hline \multicolumn{2}{|c|}{ Passive smoking $\times 40-49$ years } \\
\hline \multicolumn{2}{|c|}{ Passive smoking $\times>49$ years } \\
\hline Active smoking & $3.06(0.95,10.82)$ \\
\hline Combined smoking & $3.09(0.95,12.34)$ \\
\hline \multicolumn{2}{|l|}{ Age group } \\
\hline$<30$ years & $(-)$ \\
\hline 30-39 years & $1.55(0.83,2.95)$ \\
\hline 40-49 years & $1.77(0.94,3.39)$ \\
\hline$>49$ years & $0.74(0.34,1.59)$ \\
\hline HPV & $3.32 * *(1.90,6.09)$ \\
\hline Constant & $0.13 * *(0.06,0.29)$ \\
\hline
\end{tabular}

(B)

$(N=445)$

\begin{tabular}{cc}
\hline Smoking exposure with HPV $(+/-)$ & $(-)$ \\
No exposure $\times H P V(-)$ & $1.54(0.53,4.91)$ \\
The exposed $\times H P V(-)$ & $3.29^{*}(1.37,9.19)$ \\
No exposure $\times H P V(+)$ & $5.28^{* *}(2.25,14.52)$ \\
Passive smoking $\times H P V(+)$ & $7.47^{*}(1.83,34.03)$ \\
Active smoking $\times H P V(+)$ & $10.67^{*}(2.57,52.35)$ \\
Combined smoking $\times H P V(+)$ & $0.19^{* *}(0.071,0.42)$ \\
Constant &
\end{tabular}

(C)

$(N=420)$

\begin{tabular}{|c|c|}
\hline \multicolumn{2}{|l|}{ Smoking exposure } \\
\hline No exposure & $(-)$ \\
\hline \multicolumn{2}{|l|}{ Passive smoking } \\
\hline Passive smoking $\times<30$ years & $0.98(0.44,2.09)$ \\
\hline Passive smoking $\times 30-39$ years & $1.60(0.92,2.80)$ \\
\hline Passive smoking $\times 40-49$ years & $2.37 *(1.38,4.08)$ \\
\hline Passive smoking $\times>49$ years & $0.94(0.44,1.95)$ \\
\hline \multicolumn{2}{|l|}{$>49$ years } \\
\hline HPV & $3.51 * *(1.99,6.53)$ \\
\hline Constant & $0.18 * *(0.09,0.32)$ \\
\hline
\end{tabular}

$* p<0.05, * * p<0.001,(-)$ : negative, $(+)$ : positive. (A) Included risk factors are smoking exposure, age group, and HPV. (B) Included risk factors are HPV and the interaction between HPV and smoking exposure. (C) Included risk factors are HPV and the interaction between age group and passive smoking (excluding 25 active smokers and combined smokers).

The Interaction between Smoking Exposure and HPV To explore the combined effect of HPV and various smoking exposures on HSIL, we separated the participants into six groups. No exposure and HPV negative was the referential group; the exposed without HPV infection, no exposure but HPV positive, passive smoking with HPV positive, active smoking with HPV positive, and combined smoking with HPV positive were compared groups, shown in Table 3(B). The impact on HSIL from the three groups of smoking exposure combined with HPV positive all showed statistical significance, compared with the referential group. The OR of HPV interacting respectively with passive smoking, active smoking, and combined smoking was 5.28 (95\% CI 2.25-14.52), 7.47 (95\% CI 1.83-34.03), and 10.67 (95\% CI 2.57-52.35), indicating that smoking exposure deteriorated the effect of the HPV on patients to suffer HSIL.

The Interaction between Passive Smoking and Age Groups Since passive smoking was tested to be significant in the previous models, we continue to consider the interactive effect of passive smoking and age groups versus no exposure. As shown in Table 3(C), after excluding active smokers and combined smokers, passive smokers in 40-49 years of age and HPV infection indicated a significantly adverse impact on HSIL. The OR of 40-49-year-old passive smokers compared with that of the no exposed was 2.37 (95\% CI 1.38-4.08), reaching the highest OR among all the age groups.

Further, we considered the association between adolescent exposure history (AEH, exposure start from under 18 years of age) and HSIL. The no-exposure group was set as the reference of the odds ratios. It was found that passive smokers without AEH showed 1.58 times (95\% CI 1.02-2.45) as likely as reference to occur HSIL. Those who reported AEH showed 4.04 times (95\% CI 1.44-11.37) of a probability to suffer the disease, shown in Fig. 2. Among HSIL cases with AEH, one patient reported no more than ten years of exposure and was diagnosed CIN 2 by histological biopsy. Those persistent for 11-20 years consisted of $33.3 \%$ CIN 2, 33.3\% CIN 2/3 and 33.3\% CIN 3. Pathologists identified no CIN 2 from those with more than 20 years of exposure (Table 4 ).

\section{DISCUSSION}

Smoking has been regarded to be directly related to the incidence of cervical cancer, ${ }^{15)}$ and one of the findings is that active smokers have a higher risk of persistent HPV infection than non-smokers. ${ }^{16,17)}$ As the primary metabolite of nicotine in the human body, cotinine is used as a biomarker of tobacco exposure in the cervical mucus of active smokers. ${ }^{18)}$ Cotinine destroys DNA by activating nitrosamines, damages the local immune function, affecting the immune cytokines infiltrating upward to cervical epithelial cells, and plays a synergistic role in HPV pathogenesis. ${ }^{19-21)}$ Since cotinine can also be detected in the cervical mucus of passive smokers, researchers have suspected passive smoking leading to cervical cancer through the same pathogenesis. ${ }^{22}$

Although studies have been conducted to explore the risk factors of cervical cancer, the significant association between passive smoking and cervical lesions still remains controversial. Zeng et ll. $^{23)}$ suggested that passive smoking significantly and independently increased the risk of cervical cancer. Louie et $a{ }^{24)}$ reported that passive smoking was not an independent risk factor for the disease in the absence of active smoking. Lee et $a l .^{25)}$ demonstrated that passive smoking significantly increased the risk of CIN 2+, whereas Feng et al. ${ }^{26)}$ found no statistical association between passive smoking and CIN 2+. A nationwide survey in China ${ }^{27)}$ reported that the mortality rate of cervical cancer was higher in combined smokers than in women who were either active or passive smokers and that the risk of death increased with the daily smoking amount and accumulative years of exposure. In our study, we designed a case-control study and collected the real hospital data based on the calculated sample size. The design of this study strictly followed the STROBE statement, aiming to provide reliable 


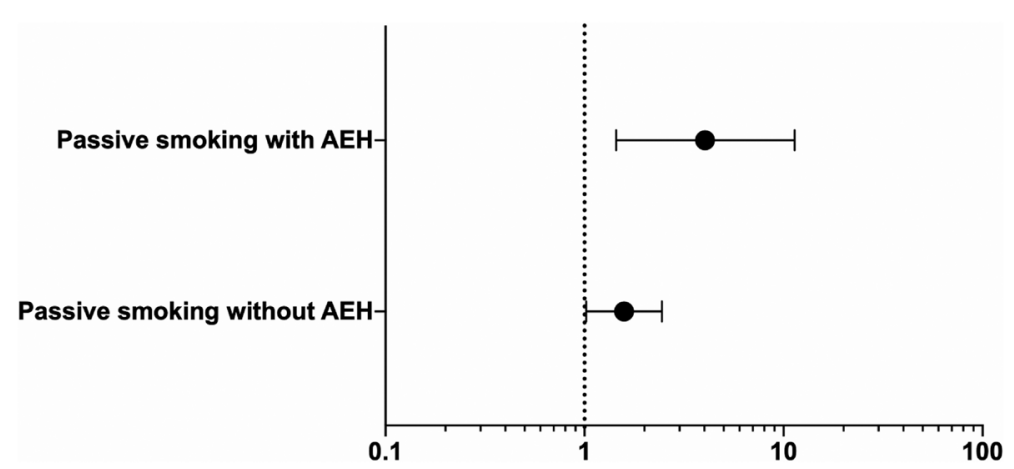

Fig. 2. Odds Ratio for HSIL in Passive Smokers with/without AEH versus No Exposure (Reference: the No-exposure Group, Odds Ratio=1.0) AEH: adolescent exposure history.

Table 4. Histopathology of HSIL Cases with Adolescent Exposure History

\begin{tabular}{|c|c|c|c|c|c|}
\hline \multirow{2}{*}{$\begin{array}{c}\text { Subjects } \\
1 .\end{array}$} & \multirow{2}{*}{$\begin{array}{c}\begin{array}{c}\text { Years } \\
\text { of age }\end{array} \\
27\end{array}$} & \multirow{2}{*}{$\begin{array}{c}\text { HPV infection } \\
\text { (+: positive, } \\
- \text { : negative) } \\
+\end{array}$} & \multicolumn{2}{|c|}{$\begin{array}{l}\text { Exposure } \\
\text { persistency } \\
\text { (years) }\end{array}$} & \multirow{2}{*}{$\begin{array}{l}\text { Histopathology } \\
\text { CIN } 2\end{array}$} \\
\hline & & & $\leq 10$ & 10 & \\
\hline 2. & 35 & + & $11-20$ & 20 & CIN 2 \\
\hline 3. & 30 & + & & 20 & CIN 2 \\
\hline 4. & 35 & + & & 18 & CIN 2/3 \\
\hline 5. & 27 & + & & 18 & CIN 2/3 \\
\hline 6. & 30 & + & & 15 & CIN 3 \\
\hline 7. & 37 & - & & 20 & $\begin{array}{l}\text { CIN } 3 \\
\text { Involving glands }\end{array}$ \\
\hline 8. & 41 & + & $21-30$ & 30 & CIN $2 / 3$ \\
\hline 9. & 35 & + & & 23 & CIN 2/3 \\
\hline 10. & 32 & + & & 27 & CIN 2/3 \\
\hline 11. & 43 & + & & 26 & $\mathrm{CIN} 2 / 3$ \\
\hline 12. & 23 & + & & 23 & CIN 3 \\
\hline 13. & 45 & + & & 29 & CIN 3 \\
\hline 14. & 47 & + & $31-40$ & 47 & CIN 2/3 \\
\hline 15. & 49 & + & $>40$ & 40 & CIN 2/3 \\
\hline
\end{tabular}

$\mathrm{HPV}$, human papillomavirus; CIN, cervical intraepithelial neoplasia.

evidence on the association between passive smoking and the other three types of exposure with HSIL. To evaluate the independent impact of passive smoking, we distinguished passive smoking from active smoking and also separated the combined effect of both passive and active smoking. The odds ratio of passive smoking relative to that of the no exposure provided the evidence that passive smoking was a risk factor of HSIL. Notably, the high exposed passive smoking experienced a higher risk than the low exposure group.

Feng et $a l .{ }^{26)}$ demonstrated that passive smoking mildly increased the risk of HPV infection. Namujju et al. ${ }^{28)}$ proved that cotinine might affect the avidity of immunoglobulin $\mathrm{G}$ induced by HPV16/18 vaccination. In our analysis, we further considered the interaction between HPV and smoking exposures and found that passive smokers with HPV positive had over five times risk than the non-smokers without HPV infection to suffer HSIL.

In the last few decades, the age of onset of cervical cancer has significantly decreased, and the proportion of cervical cancer patients under age 35 has increased from 5.01 to $9.88 \%$ of all cervical cancer patients. ${ }^{29)}$ The risk of HSIL increases at all ages. ${ }^{30,31)}$ Persistent passive smoking starting from ado- lescence may relate to the increased proportion, based on our retrospective analysis. The risk to suffer CIN 2/3 went upward along with the cumulative time of exposure. As reported, CIN 2 regression rate was $50-74 \%^{32,33)}$ as few as $32-43 \%$ of CIN 2/3 lesions will regress spontaneously without excision treatment. ${ }^{34)}$ The fact is a matter of the utmost importance to control passive smoking particularly in women and prevent exposing during juvenile.

To control recall bias, we excluded potential participants with ex-smokers or ex-passive smoking history from our study, since this form of exposure were not easy to quantify. There are also limitations to this study. The number of active smokers and combined smokers turned out to be imbalanced in the whole sample. Hence, more participants can be enrolled in future studies and give more evidence on the association of passive smoking and cervical lesions.

Our study supported that passive smoking was a significant independent risk factor on the occurrence of HSIL and showed a positive correlated dose-response relationship. HPV infection interacting with passive smoking led to an even higher risk of the disease. Middle age would increase the adverse effect of passive smoking on HSIL. Adolescent exposure to passive smoking persistent for more than 20 years would also increase the risk of HSIL.

Acknowledgments We thank Dr. Menghan Lu and Ms. Pingping $\mathrm{Yu}$ for excellent assistance for data collection. Xinxin Du is currently receiving a Grant (201508110223) from China Scholarship Council. Yi Zhou is currently receiving a Grant (201708050090) from China Scholarship Council. Hao Yang is currently receiving a Grant (2015MS0896) from the Natural Science Foundation of Inner Mongolia. Yuanguang Meng is currently receiving a Grant (81272867) from the National Natural Science Foundation of China.

Conflict of Interest The authors declare no conflict of interest.

\section{REFERENCES}

1) Torre LA, Bray F, Siegel RL, Ferlay J, Lortet-Tieulent J, Jemal A. Global cancer statistics, 2012. CA Cancer J. Clin., 65, 87-108 (2015).

2) Chen W, Zheng R, Zuo T, Zeng H, Zhang S, He J. National cancer incidence and mortality in China, 2012. Chin. J. Cancer Res., 28, 1-11 (2016)

3) Kreimer AR, Struyf F, Del Rosario-Raymundo MR, Hildesheim 
A, Skinner SR, Wacholder S, Garland SM, Herrero R, David M-P, Wheeler CM. Efficacy of fewer than three doses of an HPV-16/18 AS04-adjuvanted vaccine: combined analysis of data from the Costa Rica vaccine and PATRICIA trials. Lancet Oncol., 16, 775-786 (2015)

4) Slama J, Sehnal B, Dusek L, Zima T, Cibula D. Impact of risk factors on prevalence of anal HPV infection in women with simultaneous cervical lesion. Neoplasma, 62, 308-314 (2015).

5) Coker AL, Sanders LC, Bond SM, Gerasimova T, Pirisi L. Hormonal and barrier methods of contraception, oncogenic human papillomaviruses, and cervical squamous intraepithelial lesion development. J. Womens Health Gend. Based Med., 10, 441-449 (2001).

6) Roura E, Castellsague X, Pawlita M, et al. Smoking as a major risk factor for cervical cancer and pre-cancer: results from the EPIC cohort. Int. J. Cancer, 135, 453-466 (2014).

7) Cao S, Yang C, Gan Y, Lu Z. The health effects of passive smoking: an overview of systematic reviews based on observational epidemiological evidence. PLOS ONE, 10, e0139907 (2015).

8) Tao X, Wei L, Bo H. Survey of smoking and passive smoking status among Chinese adults in 11 provinces. Chinese Journal of Prevention and Control of Chronic Diseases, 18, 229-230 (2010).

9) Saslow D, Solomon D, Lawson HW, Killackey M, Kulasingam SL, Cain J, Garcia FA, Moriarty AT, Waxman AG, Wilbur DC, Wentzensen N, Downs LS Jr, Spitzer M, Moscicki AB, Franco EL, Stoler MH, Schiffman M, Castle PE, Myers ER. American Cancer Society, American society for colposcopy and cervical pathology, and American Society for Clinical Pathology Screening Guidelines for the prevention and early detection of cervical cancer. $C A$ Cancer J. Clin., 62, 147-172 (2012).

10) Henry MR. The Bethesda System 2001: an update of new terminology for gynecologic cytology. Clin. Lab. Med., 23, 585-603 (2003).

11) Feng X, Qian Z, Zhang B, Guo E, Wang L, Liu P, Wen X, Xu W, Jiang C, Li Y, Wu Z, Liu A. Number of cigarettes smoked per day, smoking index, and intracranial aneurysm rupture: a case-control study. Front. Neurol., 9, 380 (2018).

12) Waxman AG, Chelmow D, Darragh TM, Lawson H, Moscicki AB. Revised terminology for cervical histopathology and its implications for management of high-grade squamous intraepithelial lesions of the cervix. Obstet. Gynecol., 120, 1465-1471 (2012).

13) Darragh TM, Colgan TJ, Thomas Cox J, Heller DS, Henry MR, Luff RD, McCalmont T, Nayar R, Palefsky JM, Stoler MH, Wilkinson EJ, Zaino RJ, Wilbur DC. The Lower Anogenital Squamous Terminology Standardization project for HPV-associated lesions: background and consensus recommendations from the College of American Pathologists and the American Society for Colposcopy and Cervical Pathology. Int. J. Gynecol. Pathol., 32, 76-115 (2013).

14) Yang GH, Ma JM, Liu N, Zhou LN. Smoking and passive smoking in Chinese, 2002. Chin. J. Epidemiol., 26, 77-83 (2005).

15) Pathmanathan N, Balleine RL, Jayasinghe UW, Bilinski KL, Provan PJ, Byth K, Bilous AM, Salisbury EL, Boyages J. The prognostic value of Ki67 in systemically untreated patients with node-negative breast cancer. J. Clin. Pathol., 67, 222-228 (2014).

16) Matsumoto K, Oki A, Furuta R, Maeda H, Yasugi T, Takatsuka N, Hirai Y, Mitsuhashi A, Fujii T, Iwasaka T, Yaegashi N, Watanabe Y, Nagai Y, Kitagawa T, Yoshikawa $\mathrm{H}$. Tobacco smoking and regression of low-grade cervical abnormalities. Cancer Sci., 101, 2065-2073 (2010).

17) Alam S, Conway MJ, Chen HS, Meyers C. The cigarette smoke carcinogen benzo[a]pyrene enhances human papillomavirus synthesis. J. Virol., 82, 1053-1058 (2008).

18) Poppe WA, Peeters R, Daenens P, Ide PS, Van Assche FA. Tobacco smoking and the uterine cervix: cotinine in blood, urine and cervical fluid. Gynecol. Obstet. Invest., 39, 110-114 (1995).

19) Kjellberg L, Hallmans G, Ahren AM, Johansson R, Bergman F, Wadell G, Angstrom T, Dillner J. Smoking, diet, pregnancy and oral contraceptive use as risk factors for cervical intra-epithelial neoplasia in relation to human papillomavirus infection. Br.J. Cancer, 82, 1332-1338 (2000).

20) Eppel W, Worda C, Frigo P, Manavi M, Czerwenka K. The influence of cotinine on interleukin 6 expression in smokers with cervical preneoplasia. Acta Obstet. Gynecol. Scand., 79, 1105-1111 (2000). https://doi.org/10.1034/j.1600-0412.2000.0790121105.x

21) Szarewski A, Maddox P, Royston P, Jarvis M, Anderson M, Guillebaud J, Cuzick J. The effect of stopping smoking on cervical Langerhans' cells and lymphocytes. BJOG, 108, 295-303 (2001).

22) Prokopczyk B, Cox JE, Hoffmann D, Waggoner SE. Identification of tobacco-specific carcinogen in the cervical mucus of smokers and nonsmokers. J. Natl. Cancer Inst., 89, 868-873 (1997).

23) Zeng XT, Xiong PA, Wang F, Li CY, Yao J, Guo Y. Passive smoking and cervical cancer risk: a meta-analysis based on 3230 cases and 2982 controls. Asian Pac. J. Cancer Prev., 13, 2687-2693 (2012).

24) Louie KS, Castellsague X, de Sanjose S, Herrero R, Meijer CJ, Shah K, Munoz N, Bosch FX. Smoking and passive smoking in cervical cancer risk: pooled analysis of couples from the IARC multicentric case-control studies. Cancer Epidemiol. Biomarkers Prev., 20, 1379-1390 (2011)

25) Lee CH, Peng CY, Li RN, Chen YC, Tsai HT, Hung YH, Chan TF, Huang HL, Lai TC, Wu MT. Risk evaluation for the development of cervical intraepithelial neoplasia: development and validation of risk-scoring schemes. Int. J. Cancer, 136, 340-349 (2015).

26) Feng RM, Hu SY, Zhao FH, Zhang R, Zhang X, Wallach AI, Qiao YL. Role of active and passive smoking in high-risk human papillomavirus infection and cervical intraepithelial neoplasia grade 2 or worse. J. Gynecol. Oncol., 28, e47 (2017).

27) Jiang J, Pang H, Liu B, Nasca PC, Zhang B, Wu Y, Han W, Gates M, Lu T, Zou X, Xue F, Hou L, Wang Z, Wang Y, Chen Y, Li J. Effects of active, passive, and combined smoking on cervical cancer mortality: a nationwide proportional mortality study in Chinese urban women. Cancer Causes Control, 26, 983-991 (2015).

28) Namujju PB, Pajunen E, Simen-Kapeu A, Hedman L, Merikukka M, Surcel HM, Kirnbauer R, Apter D, Paavonen J, Hedman K, Lehtinen M. Impact of smoking on the quantity and quality of antibodies induced by human papillomavirus type 16 and 18 AS04adjuvanted virus-like-particle vaccine-A pilot study. BMC Res. Notes, 7, 445 (2014).

29) Zhang Q, Xie W, Wang F, Li RH, Cui L, Wang H, Fu X, Song J. Epidemiological investigation and risk factors for cervical Lesions: cervical cancer screening among women in rural areas of Henan Province China. Med. Sci. Monit., 22, 1858-1865 (2016).

30) Köse FM, Naki MM. Cervical premalignant lesions and their management. J. Turk. Ger. Gynecol. Assoc., 15, 109-121 (2014).

31) Hank E, Hoque ME, Zungu L. Cervical precancerous lesions and cancer among patients in the gynaecology outpatient department at a tertiary hospital in South Africa. Asian Pac. J. Cancer Prev., 14, 4903-4906 (2013).

32) Tainio K, Athanasiou A, Tikkinen KAO, Aaltonen R, Cardenas J, Hernándes, Glazer-Livson S, Jakobsson M, Joronen K, Kiviharju M, Louvanto K, Oksjoki S, Tähtinen R, Virtanen S, Nieminen P, Kyrgiou M, Kalliala I. Clinical course of untreated cervical intraepithelial neoplasia grade 2 under active surveillance: systematic review and meta-analysis. $B M J, \mathbf{3 6 0}, \mathrm{k} 499$ (2018).

33) Discacciati MG, de Souza CA, d'Otavianno MG, Angelo-Andrade LA, Westin MC, Rabelo-Santos SH, Zeferino LC. Outcome of expectant management of cervical intraepithelial neoplasia grade 2 in women followed for 12 months. Eur. J. Obstet. Gynecol. Reprod. Biol., 155, 204-208 (2011).

34) Uleberg KE, Ovestad IT, Munk AC, Brede C, van Diermen B, Gudlaugsson E, Janssen EA, Hjelle A, Baak JP. Prediction of spontaneous regression of cervical intraepithelial neoplasia lesions grades 2 and 3 by proteomic analysis. Int. J. Proteomics, 2014, 129064 (2014). 